\title{
CogWatch - Automated Assistance and Rehabilitation of Stroke-Induced Action Disorders in the Home Environment
}

\author{
Joachim Hermsdörfer ${ }^{1}$, Marta Bienkiewicz ${ }^{1}$, José M. Cogollor ${ }^{2}$, Martin Russel ${ }^{3}$, \\ Emilie Jean-Baptiste ${ }^{3}$, Manish Parekh ${ }^{3}$, Alan M. Wing ${ }^{4}$, \\ Manuel Ferre ${ }^{2}$, and Charmayne Hughes ${ }^{1}$ \\ ${ }^{1}$ Institute of Human Movement Science, Department of Sport and Health Science, \\ Technische Universität München, 80992 Munich, Germany \\ \{joachim.hermsdoerfer, marta.bienkiewicz, charmayne.hughes\} @tum.de \\ ${ }^{2}$ Centre for Automation and Robotics CAR (UPM-CSIC), Universidad Politécnica de Madrid, \\ José Gutiérrez Abascal 2, 28006 Madrid, Spain \\ $\{m$. ferre, jm. cogollor\} @upm.es \\ ${ }^{3}$ Electrical, Electronic and Computing Engineering, \\ The University of Birmingham, Birmingham, B15 2TT, U.K. \\ \{M.J .RUSSELL, EMJ198, MXP534\} @bham.ac.uk \\ ${ }^{4}$ School of Psychology, The University of Birmingham, \\ Edgbaston, Birmingham B15 2TT, UK. \\ a.m.wing@bham.ac.uk
}

\begin{abstract}
Stroke frequently causes apraxia, particularly if it affects the lefthemisphere. A major symptom of apraxia is the presence of deficits during the execution and organization of activities of daily living (ADL). These deficits may substantially limit the capacity of stroke patients to live independently in their home environment. Traditional rehabilitative techniques to improve ADL function revolve around physical and occupational therapy. This approach is labor intensive and constraints therapy to clinical environments. The CogWatch system provides an supplementary means of rehabilitation that is based on instrumented objects and ambient devices that are part of patients' everyday environment and can be used to monitor behavior and progress as well as re-train them to carry out ADL through persistent multimodal feedback.
\end{abstract}

Keywords: Apraxia, activities of daily living, rehabilitation, stroke, assistive technology.

\section{$1 \quad$ Introduction}

Following a cerebrovascular accident or stroke, a significant proportion of patients can suffer from apraxia, particularly if it affects the left-hemisphere. Major symptoms of apraxia are deficits of tool use and errors in executing and organizing action sequences of activities of daily living (ADL) [Goldenberg and Hagmann1998b;Schwartz and Buxbaum1996;Foundas et al.1995]. These deficits may substantially limit the 
capacity of stroke patients to live independently in their home environment. Effective rehabilitation is therefore a high priority, however, traditional methods are labor intensive and opportunities for patients to practice recovered skills are very limited [Smania et al. 2000;Goldenberg and Hagmann 1998a;Bowen et al. 2009].

Automated computer-based systems are therefore seen as a viable alternative. To date, the majority of computer-based rehabilitation systems is focused on treating physiological aspects of stroke, such as limb movement, and is based on robot and/or virtual environment platforms. Moreover, they require the patient to function within the system workspace rather than being adapted to the patient's normal environment. As an alternative, partners in the CogWatch Project are developing a Personal Healthcare System (PHS) that provides customized, continuous and long-term cognitive rehabilitation in the patient's familiar environment (e.g., kitchen), and allows remote monitoring of the progress of the patient by relevant clinicians.

In this study we detail the core components of the CogWatch system. The development involved the following steps: The ADL tasks relevant for patients and also suitable for automated action recognition were determined. Subsequently, clinical studies with apraxic patients were conducted in order to define typical error patterns during ADL performance. Sensors and algorithms that could provide sufficient information to recognize actions were then developed. Lastly, feedback and cueing procedures were designed to alert patients and guide error correction during task performance. Fig. 1 provides an overview of the components of the system.

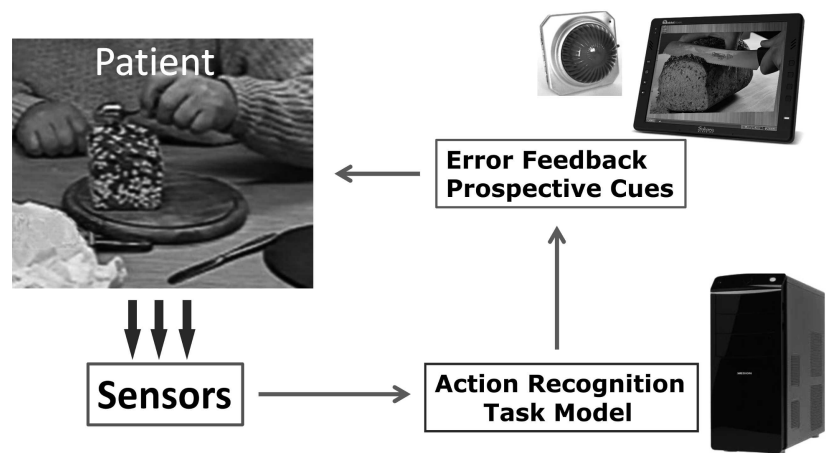

Fig. 1. Simplified schema of an automated system (CogWatch) for rehabilitation of ADL impairments following stroke

\section{ADL Task Selection}

In the first stages of CogWatch development ADL's with a clear relevance for home scenarios (from activities such as drink and meal preparation and consumption, dressing, and personal grooming) were considered for inclusion in the development. After careful consideration, the selected ADL scenario for the first prototype was a teamaking task. This task was chosen because it is highly relevant to everyday life, should be familiar to the majority of participants, is sufficiently complex to ensure the 
inclusion of a substantial number of apraxic patients, and also enables analysis about the selectivity of the effects of apraxia. Tea making has also been thoroughly studied in the literature, and thus provides a basis against which the patient results could be compared.

\section{Clinical Studies to Determine Typical Error Patterns}

We conducted experiments in a clinical setting to ascertain typical error patterns in a tea making task. To this end, we asked twenty-one apraxia patients (age $=58.10 \mathrm{y}, \mathrm{SD}=$ 13.81, 12 men, 9 women) with lesions following a single cerebrovascular accident (CVA) to prepare two cups of tea with different ingredients ("2 cup tea-making task").

Analysis indicated that patients committed errors in $48.6 \%$ of trials, with a total of 68 errors recorded. The number of errors per trial ranged from $0-10$ (mean $=3.14$, $\mathrm{SD}=2.46$ ). Consistent with previous research [Buxbaum 1998;Schwartz et al. 1998] the most frequently occurring error was that of ingredient omission (35\% of errors), with patients typically failing to put tea bags into one or both cups, or adding one or both sweetener tablets to cup2. There were also a number of trials in which apraxia patients omitted a step in the action sequence (sequence omission $=18 \%$ ), for example, turning on the empty kettle. Patients also added an unnecessary ingredient (ingredient addition $=9 \%$ ), or substituted an unnecessary ingredient for a necessary one (ingredient substitution $=9 \%$ ), carried out an action in an inappropriate way (quality $=7 \%$ ), and performed an action earlier than required (sequence anticipation $=6 \%$ ). Lastly, there was a small number of toying (4\%), misestimation (3\%), mislocation $(3 \%)$, perseveration $(3 \%)$, ingredient retraction $(2 \%)$, and perplexity errors $(2 \%)$. The data obtained from the clinical studies was used to develop the Task Models (TMs) for the first prototype (see section 5).

\section{$4 \quad$ Monitoring and Feedback Devices}

The CogWatch system is designed to be personalized to suit the needs of individual patients at the same time as being practical and affordable for home installation so that rehabilitation takes place in familiar environments performing familiar tasks. As such, it is imperative that the home-based action recognition system is affordable and easy to implement. Fortunately, emerging technologies have led to the rapid development of low-cost and easy to use monitoring and feedback devices. These devices are capable of monitoring patients' goal-oriented behavior and delivering multimodal action-guidance, and feedback to correct errors that have occurred and risk alerts to prevent accidents.

\subsection{Monitoring Devices}

Microsoft Kinect ${ }^{\mathrm{TM}}$ Sensor. The current version of the CogWatch system utilizes Kinect $^{\mathrm{TM}}$ technology to capture and record 3D hand positions and color images from 
the scene. The Kinect ${ }^{\mathrm{TM}}$ sensor is connected to a computer that interprets all signals and also allows the clinician or patient to interact with the software via a user-friendly interface (Fig. 2). This interface provides real-time visualization of the patient and image scene, as well as the signals from the sensorized objects. Furthermore, the acquired raw signals will be saved in a repository/database, and can be used for offline evaluation of patient behavior by relevant clinical personal.

The usability of the Kinect ${ }^{\mathrm{TM}}$ sensor in the CogWatch system was recently evaluated in both healthy and apraxic populations [Cogollor et al. 2012]. In that study, hand movements were recorded during a tea making task by a Kinect sensor ${ }^{\mathrm{TM}}$ and compared to data obtained by a commercially available marker-based motion capture system (CMS Zebris, Isny, Germany). The results indicated a moderate to strong correlation between signals (regardless of the neurological status of the individual), suggesting that the Kinect ${ }^{\mathrm{TM}}$ device is a reliable motion capture system in a cognitive rehabilitative context.
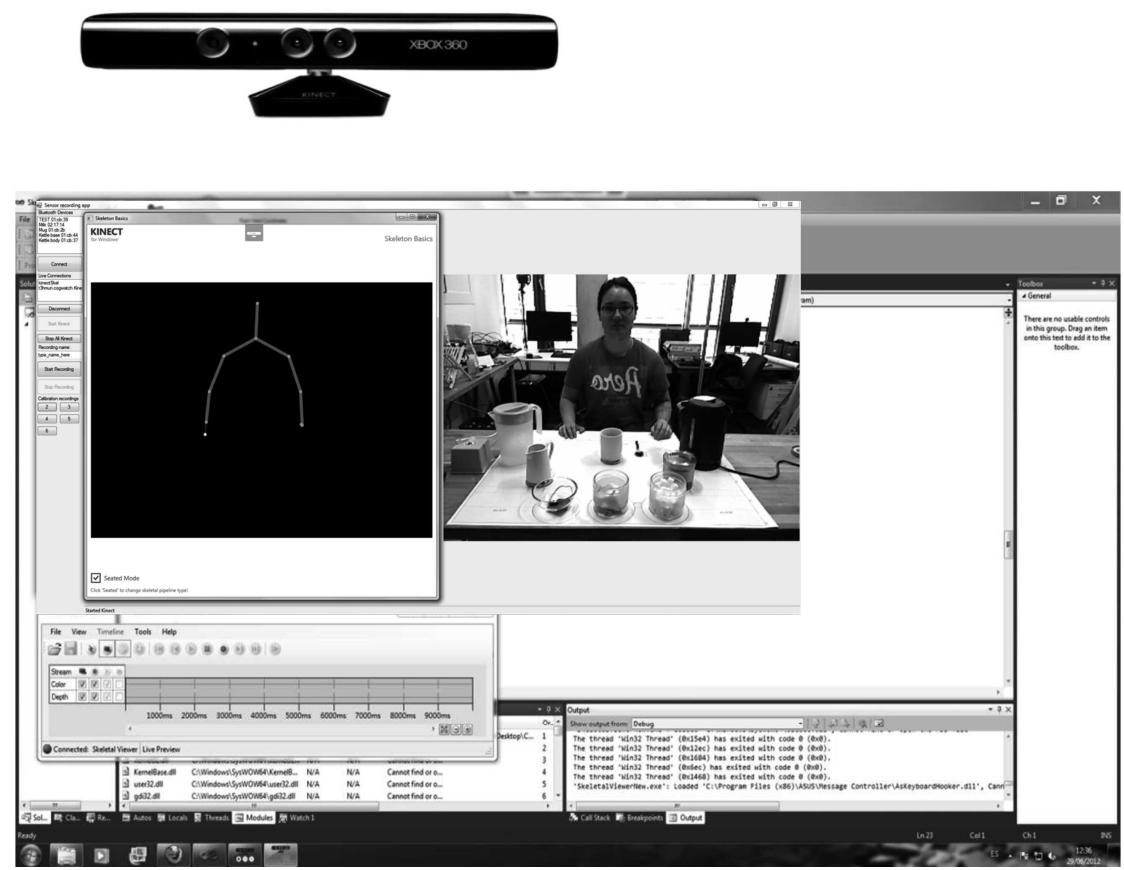

Fig. 2. Kinect ${ }^{\mathrm{TM}}$ user interface with skeleton view of arms and hands

Instrumented Objects. To detect actions at the sub-goal level the CogWatch system uses direct instrumentation of some of the objects used. A device called the CogWatch instrumented coaster (CIC) has been created to hold a 3-axis accelerometer and 3 force sensitive resistors (FSRs) in a small package that fits to the base of objects such as mugs, jugs and kettles. It is designed to be small and unobtrusive so that it does not affect the usage of the objects and has very little effect on their appearance. The CIC streams the sensor data to a host computer via Bluetooth, sampling each 
sensor at a rate of $200 \mathrm{~Hz}$ with a $12 \mathrm{bit}$ analog to digital converter. As with the $\mathrm{Ki}$ nect ${ }^{\mathrm{TM}}$, 3-D accelerometer values and the outputs of three FSRs are saved in the CogWatch system repository/database.

The accelerometer provides data that can be used to monitor changes in movement and orientation of the object that the CIC is attached to. The FSRs are used to monitor changes in weight of the object. Initial data collection has shown that these sensors are able to create very strong characterizations of actions such as pouring a kettle of water into a mug (see Fig. 3). The FSR sensors further allow the system to detect if the water is actually being poured into a mug (an example error is failure to pour into the correct container).

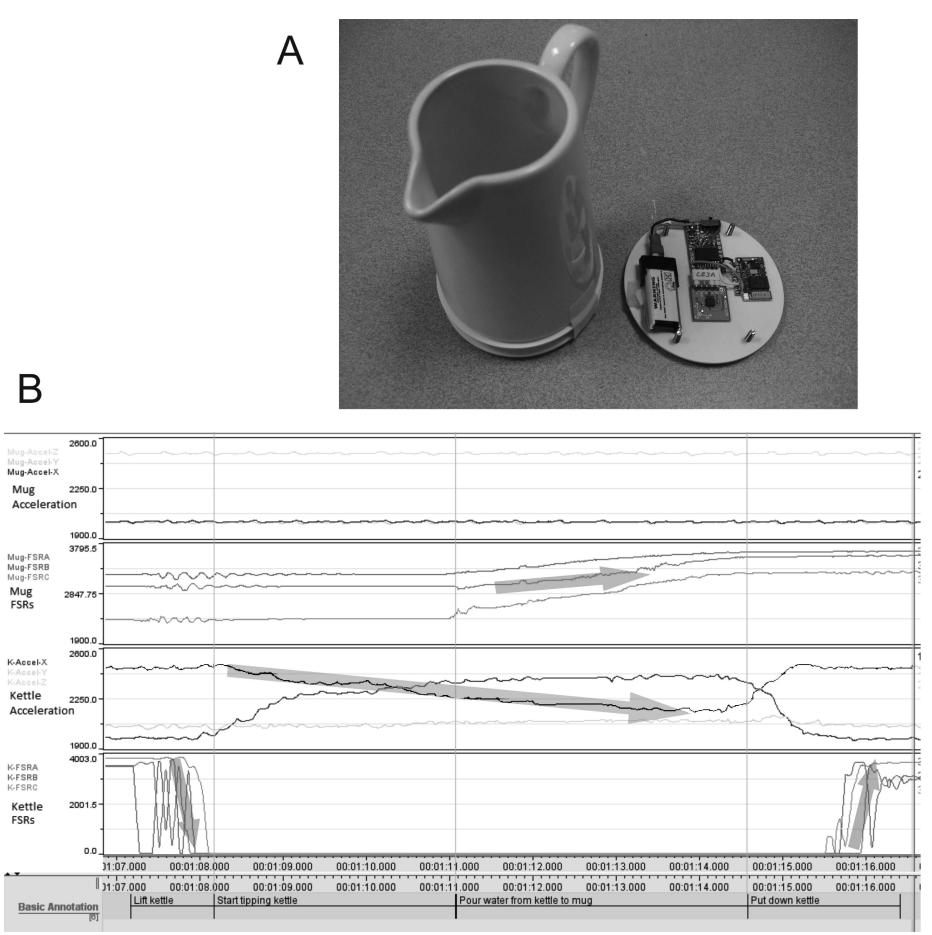

Fig. 3. CogWatch Instrumented Coasters (CIC) (A) Milk jug with fitting CIC (B) Example signals during a tea making scenario. FSRs of the kettle-CIC register lifting the kettle from the table and putting it back (see arrows $4^{\text {th }}$ panel), acceleration sensor of the kettle-CIC register kettle movement and tilt ( $3^{\text {rd }}$ panel) and FSRs of the mug-CIC register filling of the mug $\left(2^{\text {nd }}\right.$ panel).

\subsection{Feedback Devices}

Wearable Watch. A multi-functional smart watch (Meta Watch, Ltd. Dallas, Texas; see Fig. 4) is paired with the host computer via Bluetooth 4.0 technology, and is used to produce vibration signals in the event that action resulting in a safety issue has occurred. 
VTE GUI monitor. A monitor is in charge of providing Virtual Task Execution (VTE) and to provide feedback and cues to the patient during task execution (see 6 and Fig. 4). A tactile user interface (GUI) allows starting/stopping the application/simulation and selection of the task. Finally the computer is responsible for collecting all the corresponding data in the processor.

\section{$5 \quad$ Action Recognition Techniques}

- The architecture of prototype's Action Recognition Algorithm (AAR) system is defined as a set of parallel, Hidden-Markov-Model-based (HMM-based) sub-goal detectors interacting with an interpretative task model (see Fig. 4).

- The AAR interprets the sequences of measurements from the sensors (attached to the objects and tools involved in the task and to the participant's body) in terms of the actions and sub-tasks that the participant is performing. Hidden Markov Models (HMMs) were chosen for this task, because they are an appropriate technology for processing time-varying sequences of data and because many of the issues that arise in the context of the CogWatch application have already been addressed for HMMs in the context of automatic speech recognition (ASR). However, the chosen architecture of the HMM system, namely a set of parallel classifiers each modeling its own separate sub-task (defined by hierarchical task analysis) and using sub-task level HMMs, is novel from the perspective of ASR.

- A Task Model based on a Markov Decision Process (MDP) has been implemented. The states of the task model comprise sub-goals in tea making such as fill kettle, boil water, add teabag to cup, add water, stir, remove teabag, add milk, etc. The model has been tested and verified using synthetic AAR data. The notions of optimal strategy and optimal plan, which are used to define cost functions, are introduced. A shortcoming of the MDP-based approach is that it is not well-suited to dealing with ambiguity. Ambiguity arises because the AAR is imperfect and makes classification errors. A potential solution is described where the MDP is replaced by a Partially Observable MDP.

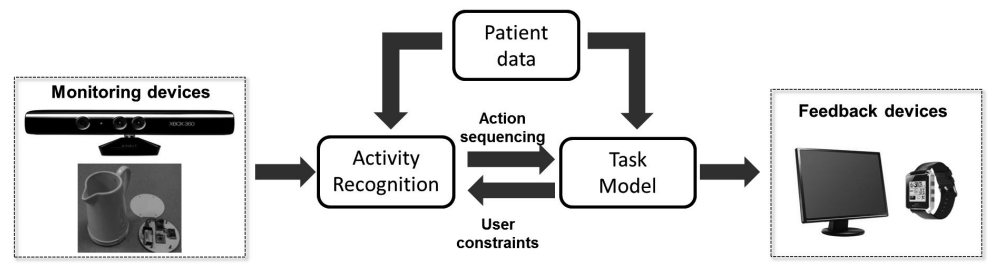

Fig. 4. Integration of Activity Recognition (AAR) and Task Models (TM) into the system

\section{$6 \quad$ Feedback and Cueing Procedures}

- Effective multimodal cues and feedback have to be investigated to determine their potential to (a) prospectively guide patients through actions to prevent the 
occurrence of apraxic errors, to (b) make patients aware of the errors when one is committed in an online fashion and to (c) make patients aware of the appropriate action. The multimodal feedback can include visual displays and markers, auditory signals, speech and gestures, vibrotactile stimulation as well as naturalistic multimodal distractors which may occur in the patients' environment (see Fig. 4 and 5).

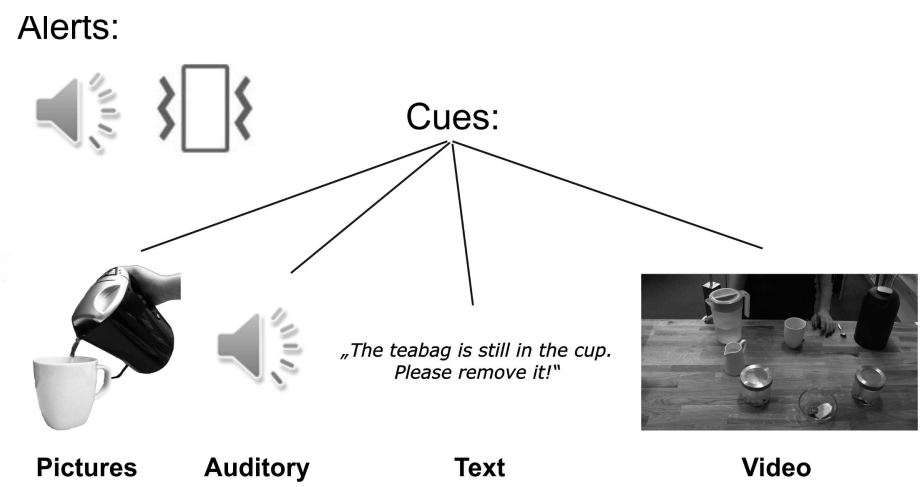

Fig. 5. Examples for feedback and type of cues available for guidance, warning and error correction

When the TM has detected an error, feedback is provided to the patient via verbal, auditory and visual (or a combination of) modalities in order to reduce the consequences of the error, prevent further errors from occurring at later stages of the task, and to preserve goal attainment if possible. Feedback information is provided in a cascading fashion, starting with simple alerts (e.g., auditory signal from a computer, vibratory signal on the wristwatch). If the patient fails to correct his or her actions, more detailed prospective cues are provided (e.g., auditory and text message, still images or videos displayed on the VTE), Feedback information can be tailored to the individual capacities of the patient who may exhibit stroke-related cognitive or language deficits. Initial evaluation of feedback alerts and information yielded good functionality, although more investigation is needed in order to clarify the most effective means of feedback for patients with different apraxic characteristics.

\section{Conclusions}

In conclusion, initial tests of an automated system to support action execution in daily life situation for apraxic patients have successfully demonstrated the feasibility of the CogWatch concept and the possibility of technical realization. The next steps include the extension of the CogWatch system to other ADL tasks, the tailoring of the tasks and system to the individual needs and abilities of the patient, and the application in home environments with affordable and failure-resistant equipment. 
Acknowledgments. The authors wish to thank all CogWatch cooperation partners for their contributions. This work has been supported by the European Commission under the grant FP7-ICT-2011-288912.

\section{References}

1. Bowen, A., West, C., Hesketh, A., Vail, A.: Rehabilitation for Apraxia. Evidence for ShortTerm Improvements in Activities of Daily Living. Stroke 40, e397 (2009)

2. Buxbaum, L.J.: Ideational apraxia and naturalistic action. Cogn. Neuropsychol. 15, 617-643 (1998)

3. Cogollor, J.M., Hughes, C.M.L., Ferre, M., et al.: Handmade task tracking applied to cognitive rehabilitation. Sensors 12, 14214-14231 (2012)

4. Foundas, A.L., Macauley, B.L., Raymer, A.M., Maher, L.M., Heilman, K.M., Rothi, L.J.G.: Ecological implications of limb apraxia: evidence from mealtime behavior. J. Int. Neuropsychol. Soc. 1, 62-66 (1995)

5. Goldenberg, G., Hagmann, S.: Therapy of activities of daily living in patients with apraxia. Neuropsychological Rehabilitation 8, 123-141 (1998a)

6. Goldenberg, G., Hagmann, S.: Tool use and mechanical problem solving in apraxia. Neuropsychologia 36, 581-589(1998b)

7. Schwartz, M.F., Buxbaum, L.J.: Naturalistic action. In: Rothi, L.J.G., Heilman, K.M. (eds.) Apraxia: The neuropsychology of Action. Lawrence Erlbaum (1996)

8. Schwartz, M.F., Montgomery, M.W., Buxbaum, L.J., et al.: Naturalistic action impairment in closed head injury. Neuropsychology 12, 13-28 (1998)

9. Smania, N., Girardi, F., Domenicali, C., Lora, E., Aglioti, S.: The rehabilitation of limb apraxia: a study in left-brain-damaged patients. Arch. Phys. Med. Rehabil. 81, 379-388 (2000) 